\title{
Macroscopic thermal entanglement in a spin chain caused by the magnetic field: Inhomogeneity effect
}

\author{
A.A. Zvyagin \\ Max-Planck-Institut für Physik Komplexer Systeme, Nöthnitzer Str., 38, D-01187, Dresden, Germany \\ B. Verkin Institute for Low Temperature Physics and Engineering of the National Academy of Sciences of Ukraine \\ 47 Lenin Ave., Kharkov 61103, Ukraine \\ E-mail: zvyagin@ilt.kharkov.ua
}

Received September 15, 2011

\begin{abstract}
The influence of the inhomogeneity on the macroscopic thermal pairwise entanglement for the system of coupled spins $1 / 2$ (qubits) has been studied. The most important effect of the inhomogeneity on the thermal entanglement is in the new role of the external potential (magnetic field), which can produce nonzero entanglement for qubits, situated not far from the inhomogeneity.
PACS: $03.65 . U d$ Entanglement and quantum nonlocality (e.g. EPR paradox, Bell's inequalities, GHZ states, etc.);
03.67.Mn Entanglement measures, witnesses, and other characterizations;
03.67.Bg Entanglement production and manipulation (for entanglement in Bose-Einstein condensates);
75.10.Pq Spin chain models.

Keywords: thermal entanglement, spin chain, impurity.

Entanglement is the fundamental aspect for a quantum many-body system, additional to correlations, which its' classical counterpart possesses [1]. It shows the nonlocal nature, in which the entangled system contains correlations that cannot be attributed to its' subsystems alone. The main subject of the theory of the quantum computation is the two-level quantum system, a qubit, which plays the role of the elementary cell containing information. It is the entanglement between qubits, which, due to the very heart of quantum mechanics $[2,3]$, permits to manipulate with many qubits simultaneously, manifesting the main advantage of a quantum computer, the quantum coherence [4]. Macroscopic entanglement demonstrates that nonlocal correlations persist even in the thermodynamic limit, where the number of particles in the system (the number of qubits in a quantum computer) tends to infinity. The majority of theoretical results for entangled multi-qubit systems was obtained for $T=0$ case [1]. However, physics of the real world ever deals with nonzero temperatures, hence, it is very important to study the thermal entanglement. The thermal entanglement for spin systems was studied recently theoretically (however, mostly for few-spin systems, not the macroscopic entanglement) [1,5]. Moreover, several experimental groups recently reported the observation of the thermal entanglement in real magnetic systems [6].
The aim of our work is to calculate analytically the characteristics of the macroscopic thermal entanglement for a model system consisting of interacting two-level subsystems (qubits) with an inhomogeneity. We attack the problem by the investigation of the thermal entanglement of a semi-infinite chain with the interaction between neighboring spins $1 / 2$, which is coupled to an impurity, described by two independent parameters. The general interest to the entanglement in one-dimensional critical systems is due to universal predictions for the scaling of the entanglement entropy [7], caused by the conformal invariance [8]. A particulary important result, which the considered model permits us to obtain, is the influence of local levels, caused by the impurity, on the thermal entanglement. This result is impossible to obtain within the boundary conformal field theory, which is mostly used for the studies of the entanglement of critical chains with impurities, or Kondo impurities in metals $[9,10]$. It is worth mentioning that the characteristics of the ground-state entanglement were calculated numerically for similar models for a finite number of qubits in Refs. 11 and 12, Ref. 13 studied the influence of a local level caused by the inhomogeneous field on the ground-state entanglement, and Ref. 14 studied the thermal entanglement of a system consisted of two qubits with an inhomogeneous field. 
In this paper we find exactly that the thermal entanglement is very sensitive to the presence of the inhomogeneity in a system of coupled qubits. The temperature range, at which one can use the quantum macroscopic entanglement between qubits is dependent on the distance to the inhomogeneity and on the applied field. We show that one can govern that range for the thermal entanglement by applying an external homogeneous potential to the system (in the case of the spin chain it is the external magnetic field). Usually it is believed that such a field destroys the quantum entanglement: Two antiferromagnetically coupled spins $1 / 2$ without the field are in the most entangled Bell (singlet) state [2], while a large field transforms their wave function to the one, which is the product of wave functions of each spin, i.e., the entanglement between those spins becomes minimal for high values of the field. Surprisingly, our exact results suggest that the field can cause the onset of a nonzero macroscopic thermal entanglement, absent without such a field.

The standard measure for the entanglement between subsystems A and B of a quantum system is the von Neumann entropy. For a subsystem $\mathrm{A} / \mathrm{B}$ it is defined as $S_{A / B}=-\operatorname{tr} \rho_{A / B} \log _{2} \rho_{A / B}$, where $\rho_{A / B}$ is the reduced density matrix for the subsystem $A(B)$. If the subsystem consists of only one qubit (spin 1/2), a simple measure for the entanglement, related to the von Neumann entropy, is the tangle [15], $\tau_{j}=4 \operatorname{det} \rho_{j}^{(1)}$, where $\rho_{j}^{(1)}=(1 / 2) \sigma_{j}^{0}+$ $+\left\langle S_{j}^{z}\right\rangle \sigma_{j}^{z}$ is the $2 \times 2$ matrix, $\sigma_{j}^{0}$ is the identity matrix, $\sigma^{z} \equiv 2 S^{z}$, and brackets denote averaging in the ground state or at nonzero temperature, so that $\tau_{j}=(1 / 4)-\left\langle S_{j}^{z}\right\rangle^{2}$. The von Neumann entropy is related to the tangle as $S^{(1)}=h[(1 / 2)(1+\sqrt{1-\tau})]$, where $h(x)=-x \log _{2} x-(1-x) \times$ $\times \log _{2}(1-x)$. However, the von Neumann entropy (and, hence, tangle) is used only if the state of the total system $\mathrm{A}+\mathrm{B}$ is pure. To analyze the pairwise entanglement, which provides more information about the quantum coherence, we need to consider the subsystem consisting of two qubits (two spins 1/2). The pairwise entanglement of two spins $1 / 2$ situated at the sites $n$ and $m$ of the quantum system can be studied using the reduced density matrix $\rho_{n m}^{(2)}$. The latter can be expressed as a $4 \times 4$ matrix $\rho_{n m}^{(2)}=$ $=\Sigma_{\mu, \nu}\left\langle S_{n}^{\mu} S_{m}^{\nu}\right\rangle \sigma_{n}^{\mu} \otimes \sigma_{m}^{v}$, where $\mu, v=0, x, y, z$, and $\otimes$ denotes the direct product. The concurrence of the entanglement of two qubits (spins 1/2) is determined [16] as $C_{n m}=\max \left(0, \mu_{1}-\mu_{2}-\mu_{3}-\mu_{4}\right)$. Here $\mu_{1,2,3,4}$ (with $\mu_{1}$ being the largest value) are the square roots of the eigenvalues of the matrix $\rho_{n m}^{(2)} \tilde{\rho}_{n m}^{(2)}$, where $\tilde{\rho}_{n m}^{(2)}$ is the spinflipped matrix of $\rho_{n m}^{(2)}, \quad \tilde{\rho}_{n m}^{(2)}=\sigma_{n}^{y} \otimes \sigma_{m}^{y}\left(\rho^{(2)}\right)^{*} \sigma_{n}^{y} \otimes \sigma_{m}^{y}$. Then the entanglement of formation is $E_{f}=h(x)$, where $x=(1 / 2)+\left[1-\left(C_{n m}^{2} / 2\right)\right]^{1 / 2} . E_{f}$ is a monotonous function of the concurrence with $E_{f}\left(C_{n m}=0\right)=0$ and $E_{f}\left(C_{n m}=1\right)=$ $=1$. This is why, for simplicity we study the concurrence in what follows. For the uniaxial spin system in the absence of the antisymmetric interactions one can take the advantage of the knowledge of the symmetries. The concurrence can be calculated using the function $C_{n m}=2 \max \left(0,|X|-\left[([1 / 4]+Z)^{2}-(1 / 4)\left(M_{n}+M_{m}\right)^{2}\right]^{1 / 2}\right)$, where for the spin model we have $M_{n}=\left\langle S_{n}^{z}\right\rangle, Z=\left\langle S_{n}^{z} S_{m}^{z}\right\rangle$, and $X=2\left\langle S_{n}^{x} S_{m}^{x}\right\rangle=2\left\langle S_{n}^{y} S_{m}^{y}\right\rangle=\left\langle S_{n}^{+} S_{m}^{-}\right\rangle=\left\langle S_{n}^{-} S_{m}^{+}\right\rangle \quad\left(S_{n}^{ \pm}=\right.$ $\left.=S_{n}^{x} \pm i S_{n}^{y} ;\left\langle S_{n}^{x} S_{m}^{y}\right\rangle=\left\langle S_{n}^{y} S_{m}^{x}\right\rangle=0\right)$.

To set the stage let us consider the Hamiltonian of the inhomogeneous semi-infinite spin $1 / 2$ chain [17]

$$
\begin{gathered}
\mathcal{H}=- \\
J \sum_{n=1}^{N-1}\left(S_{n}^{x} S_{n+1}^{x}+S_{n}^{y} S_{n+1}^{y}\right)-\hbar \gamma H \sum_{n=1}^{N} S_{n}^{z}- \\
-J^{\prime}\left(S_{0}^{x} S_{1}^{x}+S_{0}^{y} S_{1}^{y}\right)-\hbar \gamma^{\prime} H S_{0}^{z},
\end{gathered}
$$

where $J\left(J^{\prime}\right)$ describes the strength of spin-spin interactions between neighboring spins in the host chain (between the chain and impurity situated at the site 0$), \gamma\left(\gamma^{\prime}\right)$ denotes the gyromagnetic ratio for the host (impurity), $H$ is the external magnetic field, and $N$ is the number of sites in the chain (we consider $N$ odd; in the thermodynamic limit we have $N \rightarrow \infty$ ). It is known [17] that thermodynamic properties of the host chain do not depend on the sign of $J$. After the Jordan-Wigner transformation [18] $2 S_{n}^{z}=1-2 a_{n}^{\dagger} a_{n}, S_{n}^{+}=\prod_{m<n}\left(1-2 a_{m}^{\dagger} a_{m}\right) a_{n}, \quad S_{n}^{-}=$ $=a_{n}^{\dagger} \prod_{m<n}\left(1-2 a_{m}^{\dagger} a_{m}\right)$, where $a_{n}$ and $a_{n}^{\dagger}$ are Fermi operators of creation and destruction, the above Hamiltonian is exactly transformed to the quadratic form

$$
\begin{gathered}
\mathcal{H}=-\frac{J}{2} \sum_{n=1}^{N-1}\left(a_{n}^{\dagger} a_{n+1}+a_{n+1}^{\dagger} a_{n}\right)+\hbar \gamma H \sum_{n=1}^{N} a_{n}^{\dagger} a_{n}- \\
-\frac{J^{\prime}}{2}\left(a_{0}^{\dagger} a_{1}+a_{1}^{\dagger} a_{0}\right)+\hbar \gamma^{\prime} H a_{0}^{\dagger} a_{0}-\frac{\hbar H}{2}\left(N \gamma+\gamma^{\prime}\right) .
\end{gathered}
$$

Notice that the fermionic form of the Hamiltonian can (approximately) describe such inhomogeneous systems as a linear chain of Josephson junctions, cavity QED systems, linear ion traps, coupled quantum dots, etc., which were proposed to model qubits in a quantum computer [19]. This quadratic form can be diagonalized by the unitary transformation $a_{n}=\sum_{\lambda} u_{n}^{\lambda} a_{\lambda}, n=0,1, \ldots, N$. For the considered semi-infinite chain with the impurity in the thermodynamic limit we can exactly write the eigenfunctions $u_{n}^{\lambda}$ in the co-ordinate representation and eigenvalues $\varepsilon_{\lambda}$. There are two contributions. The first one is related to the continuous spectrum (in the thermodynamic limit) of the chain $\varepsilon_{k}=\hbar \gamma H-J \cos k$, with the wave functions $u_{n}^{k}=$ $=\left(2 / \pi A_{k}\right)^{1 / 2}\left[2(x+\cos k) \sin n k-I^{2} \sin ((n-1) k)\right]$ for $n \neq 0$, $u_{0}^{k}=\left(2 / \pi A_{k}\right)^{1 / 2} I \sin k$, where we denote $A_{k}=4(x+\cos k)^{2}+$ $+I^{4}-4 I^{2} \cos k(x+\cos k), I=J^{\prime} / J$ measures the relative strength of the impurity-host coupling, $x=$ $=\hbar \gamma H(\alpha-1) / J$, and $\alpha=\gamma^{\prime} / \gamma$, which measures the relative difference between the local parameter of the impurity and the host (their gyromagnetic ratios). Extended states, associated with the continuous spectrum, are present, naturally, in the homogeneous system, and the impurity renormalizes the parameters of extended states. This renormalization can be taken into account using the boundary 


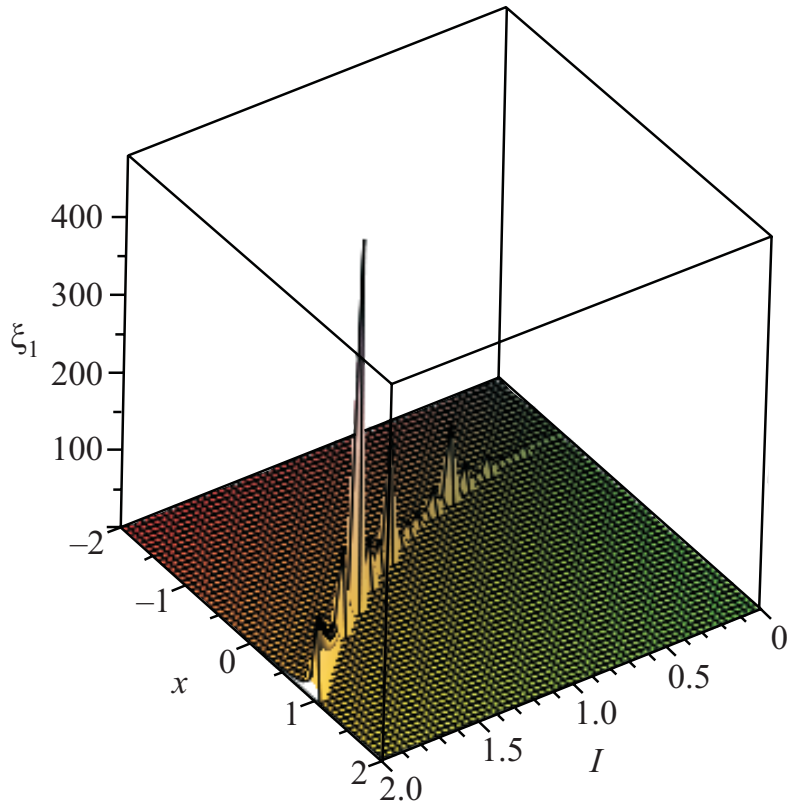

Fig. 1. (Color online) Localization length $\xi_{1}$ as the function of the relative strength of the impurity-host coupling $I=J^{\prime} / J$ and the relative strength of the magnetic field, which affects the spin of the impurity $x=\hbar\left(\gamma^{\prime}-\gamma\right) H / J$. One can see that for some values of the impurity-host stength and the field the correlation length can be of order of 500 sites of the chain.

conformal field theory. On the other hand, for $I^{2}>2 \mp 2 x$ local levels, caused by the impurity, are split off. These impurity levels have the energies $\varepsilon_{1,2}=\hbar \gamma H-$ $-J\left(r_{1,2}^{2}+1\right) / 2 r_{1,2}$, with the wave functions in the co-ordinate representation $u_{n, 1,2}=\operatorname{Ir}_{1,2}^{n}\left[\left(1-r_{1,2}^{2}\right) /\left(1+\left[I^{2}-1\right] r_{1,2}^{2}\right)\right]^{1 / 2}$, and $u_{0,1,2}=\left[\left(1-r_{1,2}^{2}\right) /\left(1+\left[I^{2}-1\right] r_{1,2}^{2}\right)\right]^{1 / 2}$, where $r_{1,2}=$ $=\left[x \pm\left(x^{2}+I^{2}-1\right)^{1 / 2}\right] /\left(I^{2}-1\right)$. Wave functions of the local states can be characterized by their localization lengths $\xi_{1,2}=-\Theta\left(I^{2}-2 \mp 2 x\right) / \ln \left(r_{1,2}\right)$, where $\Theta(a)$ is the Heaviside step function. Figure 1 shows that the localization length depends on the applied field and can be very large.

Let us denote $X=g_{n, m}$, and $M_{n}=(1 / 2)-h_{n}$. The tangle is equal to $\tau_{n}=4 h_{n}\left(1-h_{n}\right)$. Tangles for the impurity site and sites situated not very far from the impurity behave in a similar way, while for tangles for sites situated very far from the impurity the low-temperature jump at the critical value of the field is replaced by the kink, usual for the homogeneous chain, i.e., the main difference for tangles is in the ground (pure) state. It is illustrated in Fig. 2, where the field behavior of the ground-state tangles is presented. Tangles in the vicinity of the impurity show jumps in the field behavior at the critical value of the field $H_{0}=I^{2} J / 2 \hbar \gamma \sqrt{\alpha\left(I^{2}-\alpha\right)}$. Jumps are caused by the local level $\varepsilon_{1}$, which exists if the following inequality holds $I^{2}>2 \gamma^{\prime} /(1+\alpha)$. Tangles have jumps when arguments of the Heaviside functions vanish. On the other hand, at $I^{2}=2+2 x$ we have $r_{1}^{2}=1$ (no local level) and there is no jump. The value of the jump exponentially decays with

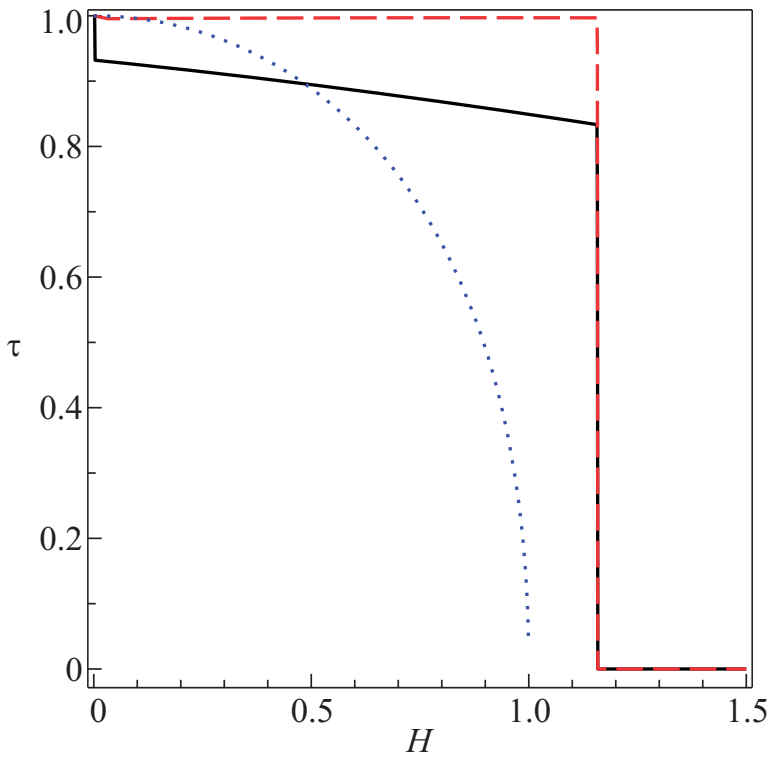

Fig. 2. (Color online) The ground-state tangles $\tau_{\mathrm{av}}$ (dotted line), $\tau_{0}$ (solid line), and $\tau_{1}$ (dashed line) as the function of the applied magnetic field $H$ (we use units in which Planck's and Boltzmann's constants, gyromagnetic ratio $\gamma$ and the exchange integral $J$ in the chain are equal to 1 for $I=2.2$ and $\alpha=1.2)$. $\tau_{0}$ and $\tau_{1}$ manifest jumps at $H_{0}=I^{2} J / 2 \hbar \gamma\left(\alpha\left(I^{2}-\alpha\right)\right)^{1 / 2}$ which take place in the region of the parameters for the impurity [20] $I^{2}>2 \gamma^{\prime} /(1+\alpha)$, as the contribution of the local level. The average tangle shows no low-temperature jump at $H_{0}$ comparing to the ones for the impurity site and near the impurity; instead $\tau_{\mathrm{av}}$ manifests the kink (due to the second order quantum phase transition) at the critical value $H_{S}=J / \hbar \gamma$, characteristic for the homogeneous system.

the distance from the inhomogeneity, and tangles for sites situated far from the impurity behave like the tangle, average over the chain (in that case we sum $h_{n}$ with respect to $n$ and divide the sum by $N, h_{\mathrm{av}}=\pi^{-1} \int_{0}^{\pi} n_{k} d k$, where $n_{k}=\left[1+\exp \left(\varepsilon_{k} / T\right)\right]^{-1}$ and we use units, for which Boltzmann's constant is equal to 1$)$ : Its field behavior reveals a kink at $H_{s}=J / \hbar \gamma$, characteristic for the second order quantum phase transition to the spin-polarized state. $\mathrm{Ob}$ viously, in the spin-polarized state $H>H_{s}$, or $H>H_{0}$, tangles are equal to zero. For comparison, in Fig. 3 we present the field dependencies of the ground-state tangles $\tau_{4}$ and $\tau_{5}$, i.e. for qubits, situated not very far from the inhomogeneity. We can see that these tangles manifest jumps at $H=H_{0}$, however, the values of tangles (except of at $H=0$ ) are much smaller than the ones near the impurity.

For any chain with interactions between only $x$ and $y$ components of neighboring spins $1 / 2$ (which Hamiltonian can be transformed to the quadratic fermion form) we can use the Wick theorem, which implies $2 Z=(1 / 2)-h_{n}-$ $-h_{m}+2 h_{n} h_{m}-2\left|g_{n, m}\right|^{2}$ for uniaxial systems without antisymmetric interactions. The concurrence in this case is 


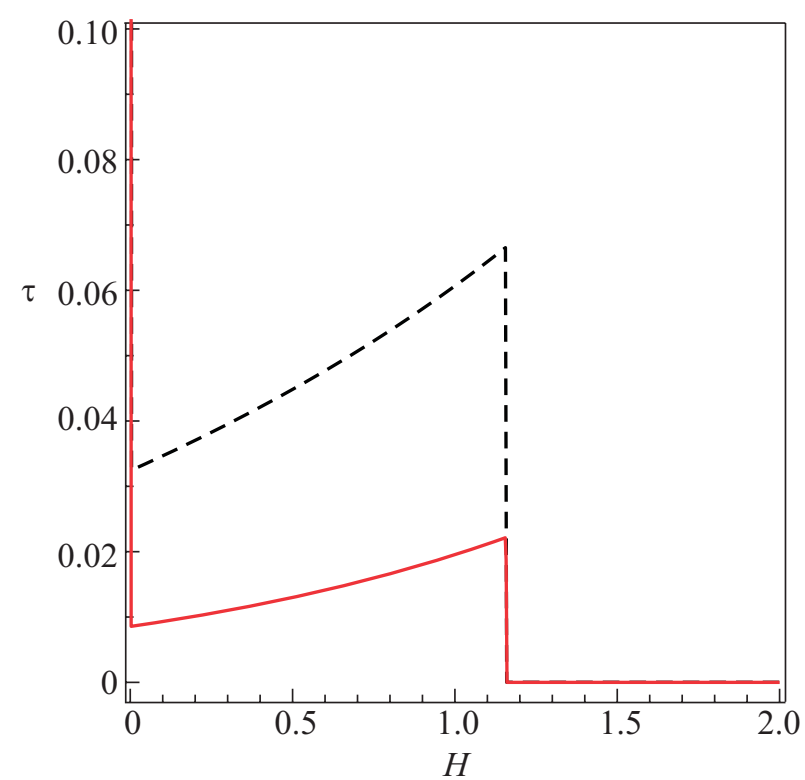

Fig. 3. (Color online) The ground-state tangles $\tau_{4}$ (dashed line), and $\tau_{5}$ (solid line) as the function of the applied magnetic field $H$ (parameters are the same as in Fig. 2). We can see the jump at $H_{0}$, however the value of tangles are much smaller than in the vicinity of the inhomogeneity.

$$
\begin{aligned}
C_{n m} & =2 \max \left(0,\left|g_{n, m}\right|-\left[\left(h_{n} h_{m}-\left|g_{n, m}\right|^{2}\right) \times\right.\right. \\
& \left.\left.\times\left(h_{n} h_{m}-\left|g_{n, m}\right|^{2}+1-h_{n}-h_{m}\right)\right]^{1 / 2}\right) .
\end{aligned}
$$

Expressions are simplified for the case of zero magnetic field $\left(h_{n}=h_{m}=1 / 2\right)$, for which we obtain $\tau_{n}=1$ and $C_{n m}=2 \max \left(0,\left|g_{n, m}\right|^{2}+\left|g_{n, m}\right|-1 / 4\right)$, i.e., the concurrence becomes nonzero for $\left|g_{n, m}\right| \leq(\sqrt{2}-1) / 2$ at $H=0$.

From now on let us concentrate on the case of the pairwise entanglement between neighboring qubits (spins 1/2). Using the values for the wave functions and the energy spectra we obtain the impurity site

$$
\begin{gathered}
h_{0}=\frac{2}{\pi} \int_{0}^{\pi} d k \frac{n_{k}}{A_{k}} I^{2} \sin ^{2} k+ \\
+\sum_{1,2} \frac{\left(1-r_{1,2}^{2}\right) n_{1,2} \Theta\left(I^{2}-2 \mp 2 x\right)}{1+\left(I^{2}-1\right) r_{1,2}^{2}} \\
g_{0,1}=\frac{2}{\pi} \int_{0}^{\pi} d k \frac{n_{k}}{A_{k}} 2 I \sin ^{2} k(x+\cos k)+ \\
+\sum_{1,2} I_{1,2} \frac{\left(1-r_{1,2}^{2}\right) n_{1,2} \Theta\left(I^{2}-2 \mp 2 x\right)}{1+\left(I^{2}-1\right) r_{1,2}^{2}}
\end{gathered}
$$

where $n_{1,2}=\left[1+\exp \left(\varepsilon_{1,2} / T\right)\right]^{-1}$. For the characteristics of sites with $n \neq 0$ we get

$$
\begin{gathered}
h_{n}=\frac{2}{\pi} \int_{0}^{\pi} d k \frac{n_{k}}{A_{k}}\left[4(x+\cos k)^{2} \sin ^{2} k n+\right. \\
\left.+I^{4} \sin ^{2}(k(n-1))-4 I^{2}(x+\cos k) \sin k n \sin (k(n-1))\right]+ \\
+\sum_{1,2} I_{1,2}^{2} r_{1,2}^{2 n} \frac{\left(1-r_{1,2}^{2}\right) n_{1,2} \Theta\left(I^{2}-2 \mp 2 x\right)}{1+\left(I^{2}-1\right) r_{1,2}^{2}} \\
g_{n, n+1}=\frac{2}{\pi} \int_{0}^{\pi} d k \frac{n_{k}}{A_{k}}\left[4(x+\cos k)^{2} \sin k n \times\right. \\
\times \sin (k(n+1))+I^{4} \sin (k(n-1)) \sin k n- \\
-2 I^{2}(x+\cos k)\left[\sin ^{2} k n+\sin (k(n+1)) \sin (k(n-1))\right]+ \\
+\sum_{1,2} I^{2} r_{1,2}^{2 n+1} \frac{\left(1-r_{1,2}^{2}\right) n_{1,2} \Theta\left(I^{2}-2 \mp 2 x\right)}{1+\left(I^{2}-1\right) r_{1,2}^{2}}
\end{gathered}
$$

We can average the concurrences for the total chain. In this situation the contributions from local levels can be neglected, and the average values are caused by only extended states: $g_{\mathrm{av}}=-\pi^{-1} \int_{0}^{\pi} \cos k n_{k} d k$. Similar behavior is revealed by qubits of the chain, situated very far from the impurity site, at distances larger than the localization lengths. Obviously, the average concurrence (and tangle) does not depend on the impurity parameters: Those contributions are of order of $N^{-1}$. The dependency of the average concurrence for the considered chain is shown in Fig. 4.

We can see that the ground-state (and low-temperature) dependence of the average tangle on the field differs from the one at the impurity site: Instead of the jump, caused by the local level, there is a kink, usual for the second order

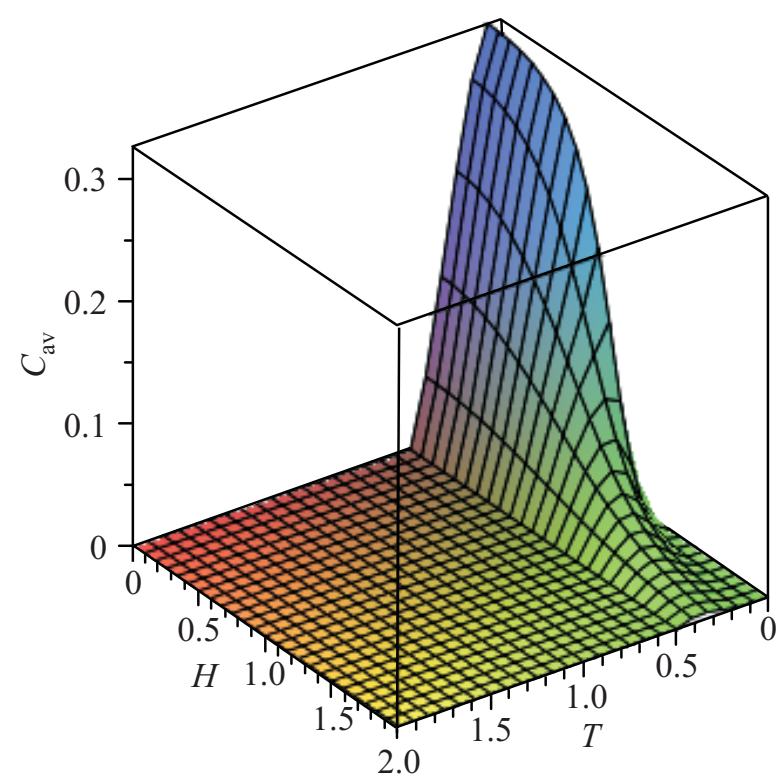

Fig. 4. (Color online) The average concurrence $C_{\mathrm{av}}$ as the function of temperature $T$ and the applied magnetic field $H$ for $J=1$. 


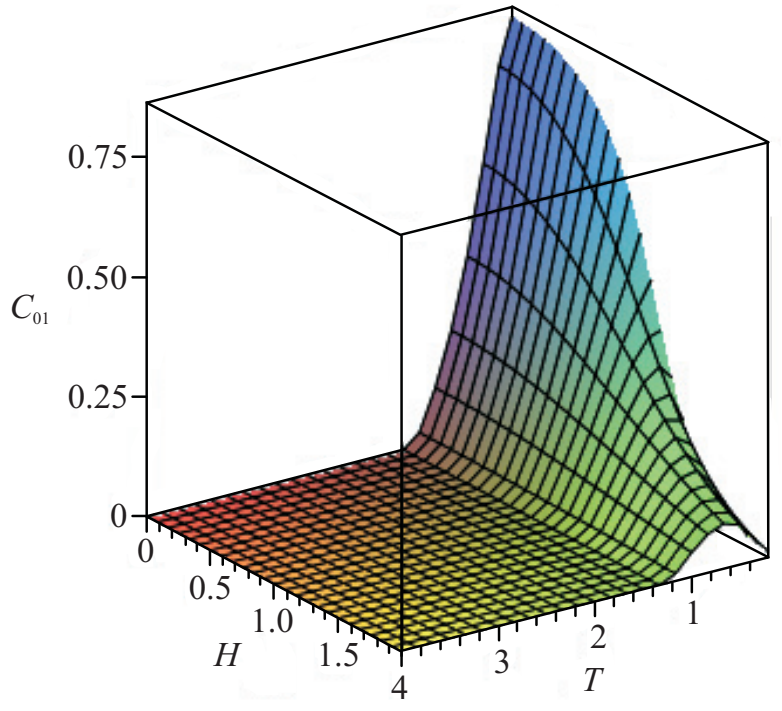

Fig. 5. (Color online) The concurrence $C_{01}$ at the impurity site as the function of temperature $T$ and the applied magnetic field $H$. Parameters are the same as in Fig. 2. The concurrence becomes smaller with the growth of the field.

quantum phase transition to the spin-polarized phase, characteristic for spin systems of such a symmetry.

Figures 5 and 6 present the temperature and magnetic field dependencies of the concurrences $C_{01}$ (near the impurity) and $C_{45}$ (several lattice sites from the impurity). One can see the drastic difference between the magnetic field behavior of concurrences at the impurity site $C_{12}$ behaves similarly, as well as the average concurrence (Fig. 4), and for sites, situated not very far from the im-

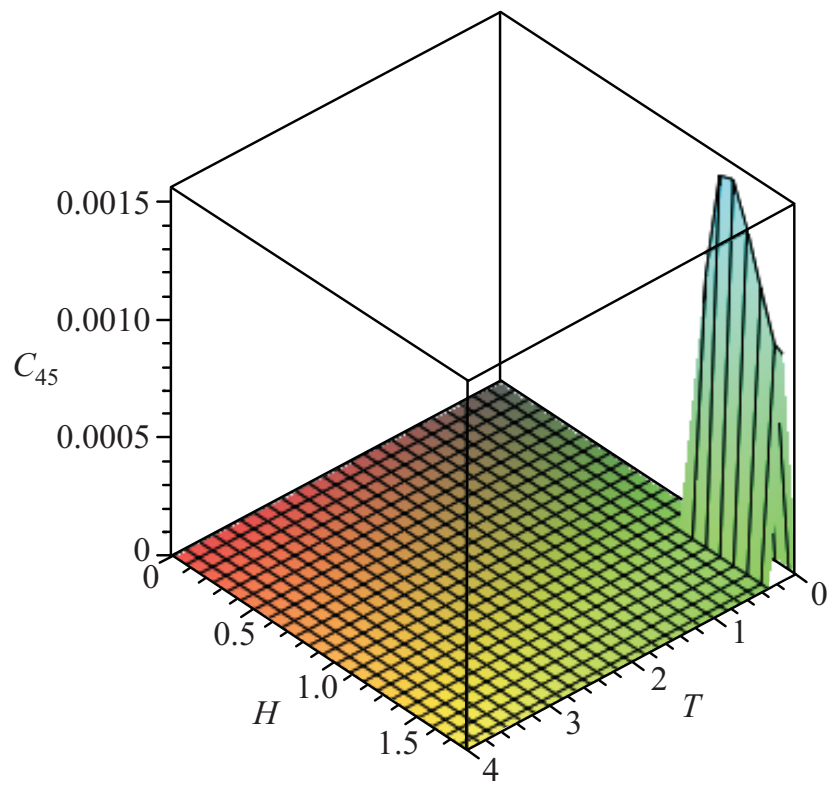

Fig. 6. (Color online) The concurrence $C_{45}$ in a short distance from the impurity as the function of temperature $T$ and the applied magnetic field $H$. Parameters are the same as in Fig. 2. Notice much smaller scale for $C_{45}$ comparing to $C_{01}$. The concurrence is zero at $H=0$ and becomes nonzero for large enough $H$. purity. The concurrence at the impurity site is maximal at zero field, and decays with the growth of the field. Pay attention that the concurrence at the impurity site is larger than the average concurrence, while the one for the sites, situated in several lattice sites from the impurity, is much smaller. The critical temperature, at which the concurrence becomes zero does not depend on $H$. Notice, however, that such critical temperatures are different for the concurrences at the impurity, nor very far from the impurity, and from the average over the chain (Fig. 4). Similar behavior (except of the scale and the low-temperature region) is manifested by average values of concurrences. In contrast, concurrences for spins, situated not very far from the impurity site, reveal a very different magnetic field behavior (cf. Fig. 6): They are zero at $H=0$, and become nonzero for large enough values of the field (of order of the coupling constant).

Hence, the pairwise thermal entanglement for neighboring qubits with inhomogeneity can be caused by the homogeneous external field. Such a behavior is very unusual. As a rule, the homogeneous external magnetic field, which does not violate the uniaxial symmetry in the spin-1/2 model of qubits is used for the initialization of qubit states to the state with the lowest entanglement. However, our results show that the same field can produce the macroscopic thermal entanglement for qubits, situated not very far from the inhomogeneity.

We have also performed the systematic study of the temperature dependencies of concurrences with a distance from the inhomogeneity at the fixed value of the field $H=0$. Figures 7 and 8 present such a dependencies as a

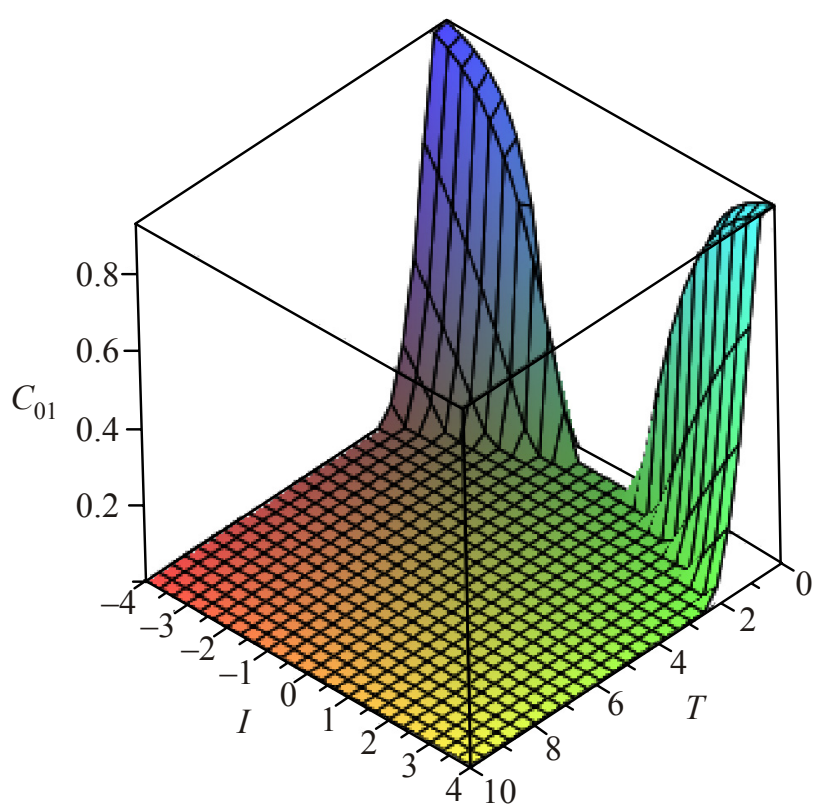

Fig. 7. (Color online) The concurrence $C_{01}$ at the impurity qubit as the function of temperature $T$ and the parameter of the inhomogeneity $I$ at zero field $H=0$. 


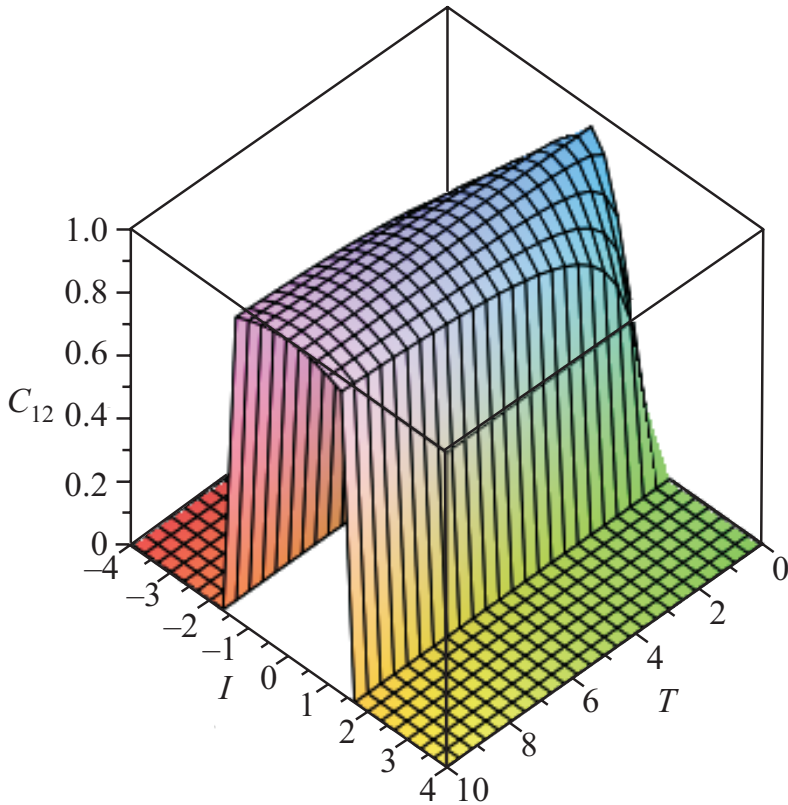

Fig. 8. (Color online) The concurrence $C_{12}$ for the nearest to the impurity qubits as the function of temperature $T$ and the parameter of the inhomogeneity $I$ at zero field $H=0$.

function of the temperature and the inhomogeneity parameter $I$ for the pairwise concurrences at the impurity qubit, $C_{01}$ and for the next to the impurity qubit $C_{12}$.

We can see a very different behavior of the pairwise concurrences as a function of the inhomogeneity parameter for $C_{01}$ and $C_{12}$ : while the pairwise concurrence for the impurity qubit is zero at small values of the impurity-host coupling, the pairwise concurrence for the qubit, situated next to the impurity, is nonzero for small values of the coupling, and becomes zero for the large values. Such a

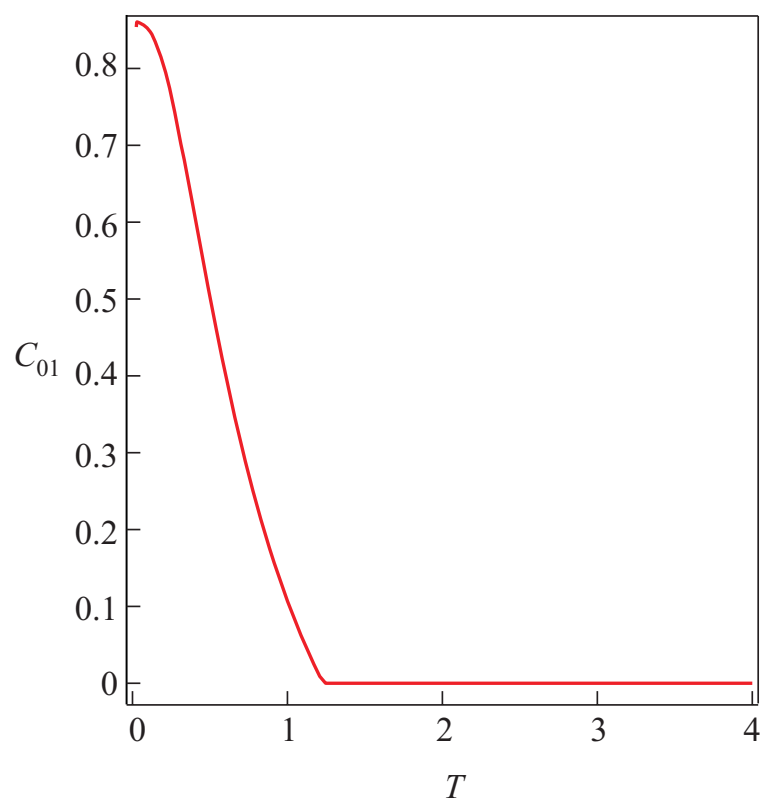

Fig. 9. (Color online) The concurrence $C_{01}$ at the impurity site as the function of temperature $T$ at the applied magnetic field $H=0.5 J / \hbar \gamma$. Parameters are the same as in Fig. 2. behavior is related to the different dependencies of considered concurrences on the coupling $I$, and, as the consequence, on the localized levels, see Eqs. (3) and (4)-(7). At the same value of the field, $H=0$, the concurrences $C_{23}$, $C_{34}$ and $C_{45}$ are zero in the same interval of parameters $T$ and $I$. For comparison, Fig. 9 shows the temperature behavior of the pairwise concurrence at the impurity qubit at $H=0.5 \mathrm{~J} / \hbar \gamma$.

Our results can be applied for the infinite chain with an impurity, by the formal substitution $I^{2} \rightarrow 2 I^{2}, n \rightarrow|n|$, hence the studied effects are caused by the impurity itself, and not by the free boundary of the considered above semiinfinite chain.

In summary, we have studied the influence of the inhomogeneity (in particular, of the local levels, caused by that inhomogeneity) on the thermal macroscopic pairwise entanglement for the system of coupled spins 1/2 (qubits) with the interactions, which preserve the uniaxial symmetry. The simple model has permitted to obtain exact analytic formulas for the characteristics of the macroscopic thermal entanglement. Our analytic $T \neq 0$ results support recent predictions based on numerical calculations mostly for finite systems, that the spatial inhomogeneity essentially affects the entanglement in real systems [10-14,21]. The most important effect of the inhomogeneity on the macroscopic thermal entanglement is in the unexpected role of the external magnetic field, which can produce nonzero entanglement for qubits, situated not very far from the inhomogeneity.

I thank MPI PKS Dresden for kind hospitality. The support from the Institute of Chemistry of V.N. Karazin Kharkov National University is acknowledged.

1. For the review use, e.g., L. Amico, R. Fazio, A. Osterloh, and V. Vedral, Rev. Mod. Phys. 80, 517 (2008) and references therein.

2. A. Einstein, B. Podolsky, and N. Rosen, Phys. Rev. 47, 777 (1935).

3. E. Schrödinger, Naturwissenschaften 23, 807 (1935).

4. M.A. Nielsen and I. Chuang, Quantum Computation and Quantum Information, Cambridge University Press, Cambridge (2000).

5. M.C. Arnesen, S. Bose, and V. Vedral, Phys. Rev. Lett. 87, 017901 (2001); X. Wang, Phys. Rev. A64, 012313 (2001); G. Töth, Phys. Rev. A71, 010301(R) (2005); A.A. Zvyagin, Phys. Rev. B80, 144408 (2009).

6. S. Ghosh, T.F. Rosenbaum, G. Aeppli, and S.N. Coppersmith, Nature 425, 48 (2003); T. Vértesi and E. Bene, Phys. Rev. B73, 134404 (2006); C. Brukner, V. Vedral, and A. Zeilinger, Phys. Rev. A73, 012110 (2006); T.G. Rappoport, L. Ghivelder, J.C. Fernandes, R.B. Guimarães, and M.A. Continentino, Phys. Rev. B75, 054422 (2007).

7. V.E. Korepin, Phys. Rev. Lett. 92, 096402 (2004).

8. P. Calabrese and J. Cardy, J. Stat. Mech. P06002 (2004). 
9. For the recent review see, e.g., I. Affleck, N. Laflorencie, and E.S. Sørensen, J. Phys. A: Math. Theor. 42, 504009 (2009) and references therein.

10. E.S. Sørensen, M.S. Chang, N. Laflorencie, and J. Affleck, J. Stat. Mech. P08003 (2007)

11. H. Fu, A.I. Solomon, and X. Wang, J. Phys. A: Math. Gen. 35, 4293 (2002).

12. X. Wang, Phys. Rev. E69, 066118 (2004).

13. T.J.G. Apollaro and F. Plastina, Phys. Rev. A74, 062316 (2006).

14. M. Asoudeh and V. Karimipour, Phys. Rev. A71, 022308 (2005).

15. V. Coffman, J. Kundu, and W.K. Wootters, Phys. Rev. A61, 052306 (2000).

16. W.K. Wootters, Phys. Rev. Lett. 80, 2245 (1998).

17. For the review see, e.g., A.A. Zvyagin, Finite Size Effects in Correlated Electron Models: Exact Results, Imperial College Press, London (2005) and references therein.
18. P. Jordan and E. Wigner, Z. Phys. 47, 631 (1928).

19. See, e.g., C.A. Sackett, D. Kielpinski, B.E. King, C. Langer, V. Meyer, C.J. Myatt, M. Rowe, Q.A. Turchette, W.M. Itano, D.J. Wineland, and C. Monroe, Nature 404, 256 (2000); A. Rauschenbeutel, G. Nogues, S. Osnaghi, P. Bertet, M. Brune, J.-M. Raimond, and S. Haroche, Science 288, 2024 (2000); M. Bayer, P. Hawrylak, K. Hinzer, S. Fafard, M. Korkusinski, Z.R. Wasilewski, O. Stern, and A. Forchel, Science 291, 451 (2001); D. Loss and E.V. Sukhorukov, Phys. Rev. Lett. 84, 1035 (2000); M.J. Hartmann, F.G.S.L. Brandao, and M.B. Plenio, Phys. Rev. Lett. 99, 160501 (2007).

20. V.Z. Kleiner and V.M. Tsukernik, Fiz. Nizk. Temp. 6, 332 (1980) [Sov. J. Low Temp. Phys. 6, 158 (1980)].

21. V.V. França and K. Capelle, Phys. Rev. Lett. 100, 070403 (2008). 